William E. Fantegrossi · Tomek Godlewski •

Rachel L. Karabenick • Jermaine M. Stephens •

Thomas Ullrich $\cdot$ Kenner C. Rice $\cdot$ James H. Woods

\title{
Pharmacological characterization of the effects of 3,4-methylenedioxymethamphetamine ("ecstasy") and its enantiomers on lethality, core temperature, and locomotor activity in singly housed and crowded mice
}

Received: 4 March 2002 / Accepted: 2 August 2002 / Published online: 1 February 2003

(C) Springer-Verlag 2003

\begin{abstract}
Rationale: Few studies have directly compared the effects of methylenedioxymethamphetamine (MDMA, "ecstasy") and its enantiomers across measures. Objectives: To investigate the capacity of MDMA and its stereoisomers to produce aggregate toxicity in mice, the influence of 5- $\mathrm{HT}_{2}$ receptors, 5-HT transporters, and ambient temperature on this effect, and to directly compare the racemate and its enantiomers in terms of their effects on core temperature and locomotor activity with and without various serotonergic pretreatments. Methods: Mice were injected with various doses of MDMA and its stereoisomers in various housing conditions, with and without pretreatments of serotonergic drugs, and at two distinct ambient temperatures; lethality was quantified $2 \mathrm{~h}$ after MDMA administration. For temperature/activity studies, mice were injected with various doses of MDMA and its enantiomers, with and without ketanserin, MDL100907, or fluoxetine pretreatments, and core temperature and locomotor activity data were collected for $24 \mathrm{~h}$. Results: Racemic MDMA and its isomers produced aggregate toxicity in mice. The lethal effects of racemic MDMA and its enantiomers were differentially attenuated by the various serotonergic pretreatments and manipulation of the ambient temperature across housing conditions. Racemic and $S(+)$-MDMA produced hyperthermic effects in mice, while $R(-)$ -
\end{abstract}

W.E. Fantegrossi $(\bowtie) \cdot$ J.H. Woods

Department of Pharmacology, University of Michigan,

Medical School, 1301 MSRB III, Ann Arbor, MI 48109-0632, USA

e-mail: billfan@umich.edu

Fax: +1-734-7647118

T. Godlewski · R.L. Karabenick · J.M. Stephens · J.H. Woods Department of Psychology (Biopsychology Program),

University of Michigan, Ann Arbor, MI 48109-1109, USA

T. Ullrich · K.C. Rice

Laboratory of Medicinal Chemistry, NIDDK,

National Institutes of Health, Building 8, Room B1-21, Bethesda, MD 20892, USA
MDMA did not. The pretreatment drugs attenuated the hyperthermic effects of racemic MDMA, but were less effective in blocking $S(+)$-MDMA-induced hyperthermia. Racemic MDMA and both enantiomers stimulated locomotor activity, although $R(-)$-MDMA was least effective. The pretreatments all reduced the locomotor stimulant effects of racemic MDMA but potentiated $S(+)$-MDMAinduced hyperlocomotion. Conclusions: The MDMA isomers have heterogeneous effects that can be demonstrated across a wide range of endpoints.

Keywords MDMA · Aggregate toxicity - 5-HT . Thermoregulation · Hyperthermia

\section{Introduction}

The term "aggregate toxicity" refers to the finding that many drugs exhibit an increase in their actions when administered to animals housed together. Perhaps the first insights into the characterization of this effect occurred when Gunn and Gurd (1940) observed in their studies on benzedrine and other stimulants that "the symptoms of excitement are much more pronounced if several injected animals are kept together in a cage or tray." Later studies conducted by Chance and colleagues produced the first empirical evidence that aggregation potentiates the lethal effects of stimulants (Chance 1947). Since these initial characterizations of the phenomenon, aggregate toxicity in rodents (here defined as at least a halving of the $\mathrm{LD}_{50}$ ) has been noted for many other drugs, including caffeine, amiphenizole, pipradrol, $\beta$-phenylisopropylhydrazine, methylphenidate and methamphetamine (Greenblatt and Osterberg 1961); isocarboxazide, desmethylimipramine, naloxone, and pethidine (Doggett et al. 1977); morphine (Mohrland and Craigmill 1980); racemic methylenedioxyamphetamine (MDA) and, most germane to the 
present research, racemic methylenedioxymethamphetamine (MDMA, "ecstasy"; Davis and Borne 1984).

Investigations into the aggregate toxicity of MDMA may be particularly relevant given the typical "rave" environment in which the drug is often abused by humans. At these all-night dance parties, conditions of crowding and high ambient temperature are common (Green et al. 1995) and may act to potentiate the toxic effects of MDMA. Indeed, an initial report on the lethal effects of MDMA in the human noted that "In almost every case, a recreational dose of the drug had been taken at a dance club or party where crowds danced vigorously" (Randall 1992).

The hyperthermic effects of MDMA in rodents were first described by Nash et al. (1988) and, since then, experimental attempts to identify the potential mechanisms of MDMA-induced toxicity and neurodegeneration have been complicated by the observation that these effects appear to be tightly interwoven with MDMAinduced hyperthermia (Broening et al. 1995). Additionally, the protective effects of several compounds against MDMA-induced neurodegeneration have been attributed to their effectiveness in inducing hypothermia on their own (Farfel and Seiden 1995; Malberg et al. 1996) or in blocking MDMA-induced hyperthermia (Colado et al. 1998). Further studies have also demonstrated protection against MDMA-induced neurotoxicity by lowering core temperature via non-pharmacological means, including restraint stress (Miller and O'Callaghan 1994) and manipulation of the ambient temperature (Malberg and Seiden 1998).

Despite this widespread interest in the relationship between temperature and MDMA-induced neurodegeneration, no widely accepted mechanism for the hyperthermic effects of MDMA has yet been described. However, the 5- $\mathrm{HT}_{2 \mathrm{~A} / 2 \mathrm{C}}$ agonist 1-(4-iodo-2,5-dimethoxyphenyl)-2aminopropane (DOI) and the $5-\mathrm{HT}_{2 \mathrm{C}}$ agonist $m$ chlorophenylpiperazine (M-CPP) have both been shown to induce hyperthermia (Mazzola-Pomietto et al. 1997), and, in addition to releasing 5-HT (Rudnick and Wall 1992), MDMA has also been shown to act as an agonist at these receptors (Nash et al. 1994). Additionally, we (Ullrich et al. 2000) have previously shown that both the $5-\mathrm{HT}_{2 \mathrm{~A} / 2 \mathrm{C}}$ antagonist ketanserin, and the selective $5-\mathrm{HT}_{2 \mathrm{~A}}$ antagonist MDL100907 produce dose-dependent hypothermic effects in mice, further implicating the $5-\mathrm{HT}_{2}$ receptor family in rodent thermoregulation.

No recent studies have examined the effects of MDMA in crowded animals, and, to our knowledge, the effects of the MDMA stereoisomers on temperature, locomotor activity, and lethality have never been directly compared in any housing condition. We were therefore interested in investigating the capacity of racemic MDMA and its enantiomers to produce aggregate toxicity in mice. Further experiments were conducted to study the influence of ambient temperature, the 5- $\mathrm{HT}_{2 \mathrm{~A} / 2 \mathrm{C}}$ antagonist ketanserin, the selective 5- $\mathrm{HT}_{2 \mathrm{~A}}$ antagonist MDL100907, and the serotonin selective re-uptake inhibitor (SSRI) fluoxetine on MDMA-induced lethality across isomers and housing conditions. Additionally, temperature and locomotor activity dose-effect curves were generated for racemic MDMA and its stereoisomers using implanted radiotelemetery probes in order to directly compare the enantiomers and correlate lethal doses with these physiological and behavioral measures. Pretreatments that proved effective in blocking lethality were then assessed alone, or in combination with MDMA, for their effects on core temperature and locomotor activity.

\section{Methods}

\section{Animals}

Male NIH Swiss mice (Harlan Sprague Dawley Inc., Indianapolis, Ind.) weighing $20-30 \mathrm{~g}$ were housed 12 animals per $20.32 \times 30.48 \times 12.70-\mathrm{cm}$ Plexiglas cage in a temperature-controlled room that was maintained at an ambient temperature of $22 \pm 2^{\circ} \mathrm{C}$ at 45-50\% humidity. Lights were set to a 12-h/12-h light/dark cycle. Animals were fed Lab Diet rodent chow (Laboratory Rodent Diet \#5001, PMI Feeds, Inc., St. Louis, Mo.) and water ad libitum until immediately before testing. Animals were not used in experiments until at least 2 days after arrival in the laboratory. Each animal was used only once, and was sacrificed immediately after use. Studies were carried out in accordance with the Declaration of Helsinki and with the Guide for Care and Use of Laboratory animals as adopted and promulgated by the National Institutes of Health. The experimental protocol was approved by the University of Michigan's University Committee on the Use and Care of Animals.

Procedure

Aggregate toxicity experiments

On experimental days, mice were weighed, marked, and returned to the home cage. MDMA doses were then calculated and prepared for injection. Animals were subsequently removed from the home cage, injected intraperitoneally (i.p.) and placed into $15.24 \times 25.40 \times 12.70-\mathrm{cm}$ Plexiglas mouse cages singly, in groups of 6, or in groups of 12. All aggregation groups were drawn from the same home cage in order to minimize aggression (indeed, none was observed during the conduct of these experiments). Except where stated, aggregate toxicity experiments were conducted in the colony room at an ambient temperature of $22 \pm 2^{\circ} \mathrm{C}$. Neither food nor water were available during the aggregate toxicity tests. Mice were observed for $2 \mathrm{~h}$ post-injection, and lethality was quantified at the end of this period. No mice were removed from their respective cages before the expiration of the 2-h test period. The lethal effects of all doses of all MDMA compounds were doubly determined for each caging condition - thus, all statistics were performed with $n=2$ observations. For the singly housed mice, two separate groups of 6 mice (a total of 12 mice) were studied - lethality was computed for each group, then averaged and SEM computed. The same was true for the mice housed 6 per cage. For the mice housed 12 per cage, two separate groups of 12 mice (a total of 24 mice) were studied lethality was computed for each group, then averaged and SEM computed. Wherever possible, MDMA doses were not increased past the experimentally determined $\mathrm{LD}_{75}$ for each compound.

\section{Ketanserin, MDL100907, and fluoxetine antagonism experiments}

Doses of racemic MDMA and its stereoisomers that produced equivalent lethality in the previous experiments were studied with and without a pretreatment of the $5-\mathrm{HT}_{2 \mathrm{~A} / 2 \mathrm{C}}$ antagonist ketanserin $(5.6 \mathrm{mg} / \mathrm{kg})$, the selective 5-HT $2 \mathrm{~A}$ antagonist MDL100907 $(1.0 \mathrm{mg} /$ $\mathrm{kg})$, or the SSRI fluoxetine $(10 \mathrm{mg} / \mathrm{kg})$ in the two most extreme 
housing conditions ( 1 and 12 mice per cage). All pretreatments were administered i.p. $15 \mathrm{~min}$ prior to MDMA injection. To minimize the need for interpolation, the $\mathrm{LD}_{75}$ for each compound was used; however, in singly housed mice receiving racemic MDMA, it was impossible to experimentally determine an $\mathrm{LD}_{75}$ due to the extreme slope of the lethality curve. During these experiments, mice were weighed, marked, and returned to the home cage while MDMA and pretreatment doses were calculated and prepared for injection. All other procedures were as previously described.

\section{Cold-room protection experiments}

To assess the effects of ambient temperature on MDMA-induced lethality, the $\mathrm{LD}_{75}$ for racemic MDMA and its stereoisomers were studied in a $4^{\circ} \mathrm{C}$ cold room, in singly housed mice and in mice housed 12 per cage. All other procedures were as previously described.

\section{Core temperature and locomotor activity experiments}

Following appropriate anesthetization with ketamine $(100 \mathrm{mg} / \mathrm{kg}$, i.p.) and xylazine (10 mg/kg, i.p.), the abdominal area of each mouse was shaved and sanitized with iodine swabs. A rostralcaudal cut approximately $1.5 \mathrm{~cm}$ in length was made with skin scissors, providing access to the intraperitoneal cavity. A cylindrical glass-encapsulated radiotelemetry probe (model ER-4000 EMitter, Mini Mitter, Bend, Ore., USA) was then inserted, and the incision was closed using absorbable 5-0 chromic gut suture material. Surgeries were carried out at least 7 days before initiation of experimental conditions, allowing time for incisions to heal and for the mice to recover normal body weights. The transmitters produced activity- and temperature-modulated signals, which were sent to a receiver (model ER-4000 Receiver, Mini Mitter Co., Inc.) underneath each mouse cage. Temperature and locomotor activity data were thus collected from singly housed mice at 5-min intervals and processed simultaneously by the Vital View data acquisition system (Mini Mitter Co., Inc.).

\section{Data analysis}

Data from the lethality experiments are presented as mean \pm SEM and were compared using Fisher's exact test. For the core temperature and locomotor activity experiments, data are presented as mean \pm SEM and were compared to minimum and maximum values obtained from equivolume saline controls $(n=6$, data not shown) using Student's one-sample $t$-tests. All statistical tests were performed using commercially available software, and significance was judged at $P<0.05$.

Drugs

Racemic MDMA and its stereoisomers were supplied by the National Institute on Drug Abuse (Research Technology Branch, Research Triangle Park, N.C.) and were dissolved in physiological saline prior to injection. Ketanserin tartrate was purchased from Research Biochemicals International (Natick, Mass.), and dissolved in sterile water and 5\% dimethyl sulfoxide (DMSO). MDL100907 was synthesized at the Laboratory of Medicinal Chemistry at the National Institutes of Diabetes, Digestive and Kidney Disorders at the National Institutes of Health (Bethesda, Md.) and dissolved in sterile water and $0.5 \mathrm{~N} \mathrm{HCl}$. Fluoxetine was obtained from Eli Lilly, Inc. (Indianapolis, Ind.) and dissolved in physiological saline. All injections were administered i.p. in a volume equal to body weight $(\mathrm{g}) / 100$

\section{Results}

\section{Aggregate toxicity experiments}

Increasing cage density potentiated the lethal effects of racemic MDMA and the stereoisomers. Racemic MDMA and $S(+)$-MDMA were approximately equipotent in terms of their lethal effects across all three cage conditions, while $R(-)$-MDMA was approximately half as potent (Fig. 1). Deaths typically occurred within $30 \mathrm{~min}$ post-
Fig. 1 Top two, and bottom left panels Lethality curves across three housing conditions for racemic methylenedioxymethamphetamine (MDMA) and its stereoisomers in mice. Abscissa Dose $(\mathrm{mg} / \mathrm{kg})$ of MDMA. Ordinate Percentage lethality assessed at $2 \mathrm{~h}$ after injection. Bottom right panel Potentiation of the lethal effects of MDMA and its enantiomers due to increased cage density. Abscissa Number of mice per cage. Ordinate Interpolated $\mathrm{LD}_{50}$
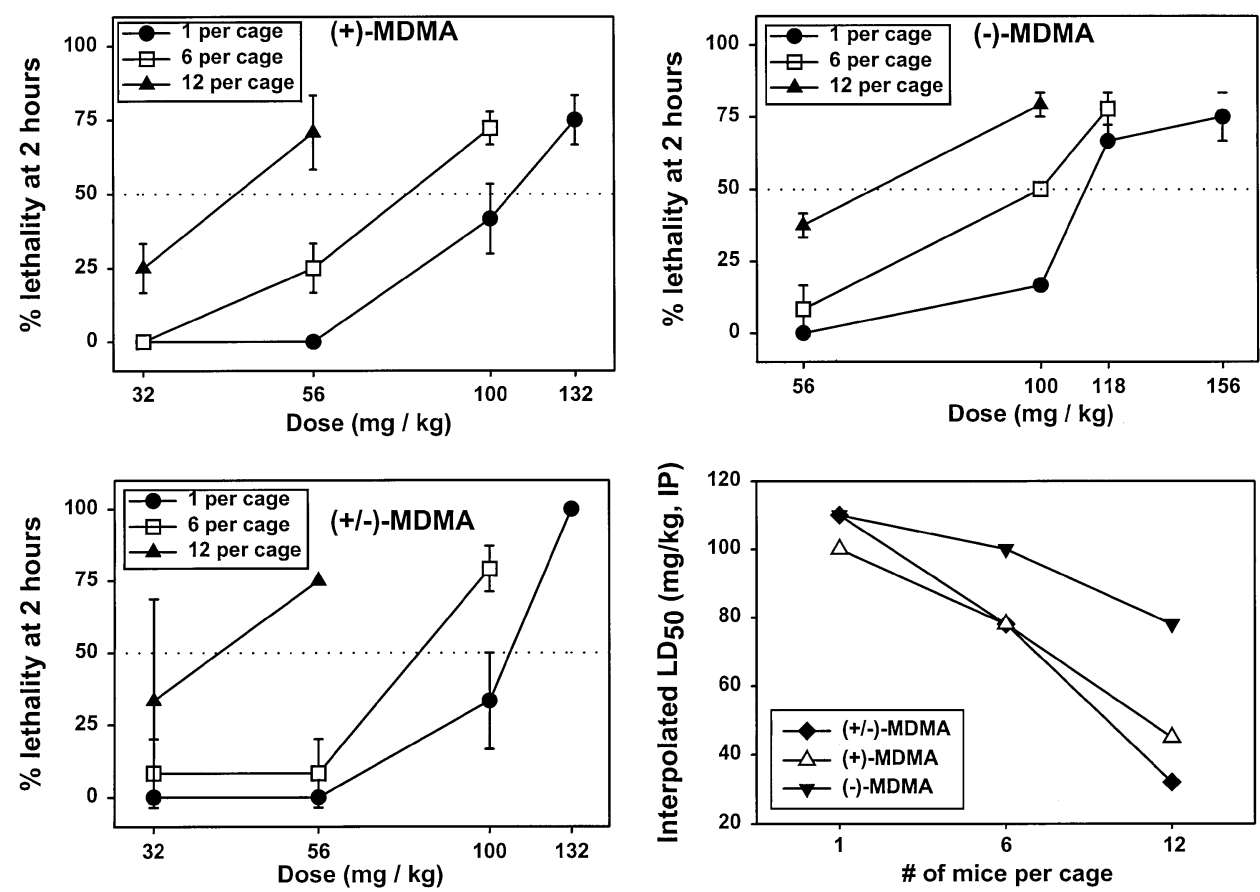

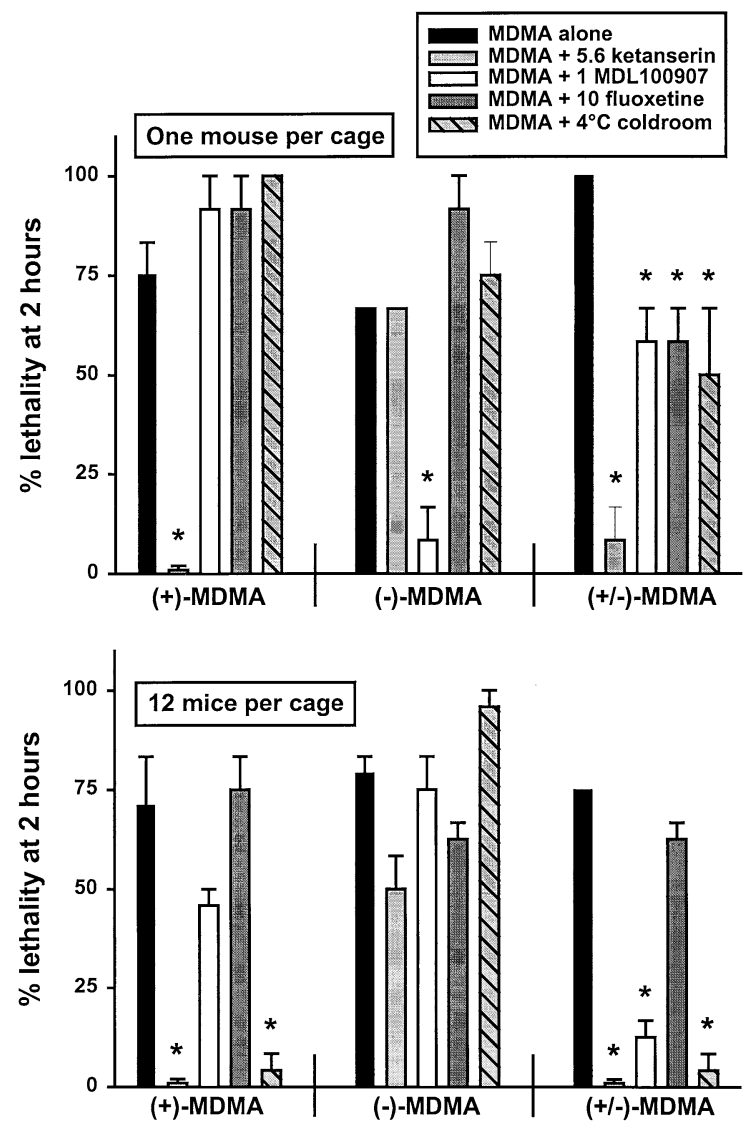

Fig. 2 Lethality-matched $\left(\mathrm{LD}_{75}\right)$ doses of racemic methylenedioxymethamphetamine (MDMA) and its stereoisomers with and without ketanserin, MDL100907, or fluoxetine pretreatment in singly housed mice (top) and in mice housed 12 per cage (bottom). Each bar represents the mean \pm SEM (based on 12 mice for the singly housed group and 24 for the 12 mice-per-cage group). Abscissae Caging condition and dose $(\mathrm{mg} / \mathrm{kg})$. Ordinates Percentage lethality assessed at $2 \mathrm{~h}$ after injection. Asterisks indicate significance $(P<0.05)$ by the Fisher's exact test

injection, and all mice surviving to 60 min post-injection subsequently survived the final hour of testing. Interpolating the $\mathrm{LD}_{50}$ for each compound at each housing condition allows a comparison of the potentiation of lethality produced by aggregation (Fig. 1, bottom right). Plotted in this manner, the lethal effects of racemic and $S(+)$-MDMA show a greater potentiation due to crowding (indicated by a steeper slope) than do the lethal effects of $R(-)$-MDMA.

Ketanserin, MDL100907, and fluoxetine antagonism experiments

Pretreatment with ketanserin completely blocked the lethal effects of racemic MDMA $(P<0.05)$ and $S(+)$ MDMA $(P<0.05)$ in singly housed and crowded mice; however, the lethal effects of $R(-)$-MDMA were not affected in either housing condition (Fig. 2). In singly housed mice, MDL100907 attenuated the lethal effects of racemic MDMA $(P<0.05)$ and almost entirely blocked the lethal effects of $R(-)$-MDMA $(P<0.05)$, but did not alter $S(+)$-MDMA-induced lethality. Interestingly, MDL100907 only attenuated the lethal effects of racemic MDMA in the aggregate housing condition $(P<0.05)$. Fluoxetine pretreatment attenuated the lethal effects of racemic MDMA $(P<0.05)$ in singly housed but not crowded mice, and the lethal effects of the enantiomers were not altered by this pretreatment in either housing condition.

\section{Cold-room protection experiments}

The cold ambient environment significantly attenuated the lethal effects of racemic MDMA in singly housed mice $(P<0.05$; Fig. 2$)$; however, the lethal effects of the stereoisomers were not affected in this housing condition. Interestingly, the cold ambient environment completely abolished the lethal effects of racemic MDMA $(P<0.05)$ and $S(+)$-MDMA $(P<0.05)$ in mice housed 12 per cage, while the lethal effects of $R(-)$-MDMA remained unchanged.

\section{Core temperature and locomotor activity experiments}

Racemic MDMA and $S(+)$-MDMA produced a dose- and time-dependent hyperthermic effect in mice (Fig. 3, bottom and top left panels, respectively). For these compounds, the duration of hyperthermia was approximately $2 \mathrm{~h}$, and the peak body temperature reached was approximately $41^{\circ} \mathrm{C}$. By $3 \mathrm{~h}$ post-injection, body temperature had returned to normal and, from this point on, did not deviate outside the normal temperature range for the remainder of the 24-h sampling period (data not shown). For both racemic MDMA and $S(+)$-MDMA, significant hyperthermia was produced at $32 \mathrm{mg} / \mathrm{kg}$ - a sublethal dose in singly housed animals. However, several deaths occurred in the 56-mg/kg $S(+)$-MDMA group (indicated by the arrows on Fig. 3, top left panel), although no deaths occurred at this dose (or any other) with $S R(+/-)$-MDMA. Interestingly, no dose of $R(-)$-MDMA produced any measurable effects on body temperature (Fig. 3, top right panel). Doses of $R(-)$-MDMA higher than $100 \mathrm{mg} / \mathrm{kg}$ were not tested due to the lethal effects of these doses in previous experiments.

The various pretreatment drugs used in the previous lethality studies were also assessed for their effects on body temperature. Although MDL100907 $(1 \mathrm{mg} / \mathrm{kg})$ and fluoxetine $(10 \mathrm{mg} / \mathrm{kg})$ did not significantly increase or decrease core temperature, ketanserin $(5.6 \mathrm{mg} / \mathrm{kg})$ produced a dramatic hypothermia (Fig. 3, bottom right panel). The ketanserin-induced hypothermia lasted approximately $2.5 \mathrm{~h}$ and dropped core temperature to approximately $32^{\circ} \mathrm{C}$. By $3 \mathrm{~h}$ post-injection, ketanserintreated animals had returned to the range of normal body temperature and did not deviate outside this range from 
Fig. 3 Effects of racemic methylenedioxymethamphetamine (MDMA), its stereoisomers, and the various serotonergic pretreatments on core temperature in singly housed mice. Each point represents the mean \pm SEM $(n=6$ mice per dose). Abscissae Time after injection (hours). Ordinates

Core temperature $\left({ }^{\circ} \mathrm{C}\right)$. The defined region between approximately $36^{\circ} \mathrm{C}$ and $38^{\circ} \mathrm{C}$ represent the normal range of rodent core temperature measured over a 24-h period following equivolume saline injection. Arrows in top left panel indicate time of death of one mouse per arrow following injection of $56 \mathrm{mg} / \mathrm{kg}$ $S(+)$-MDMA
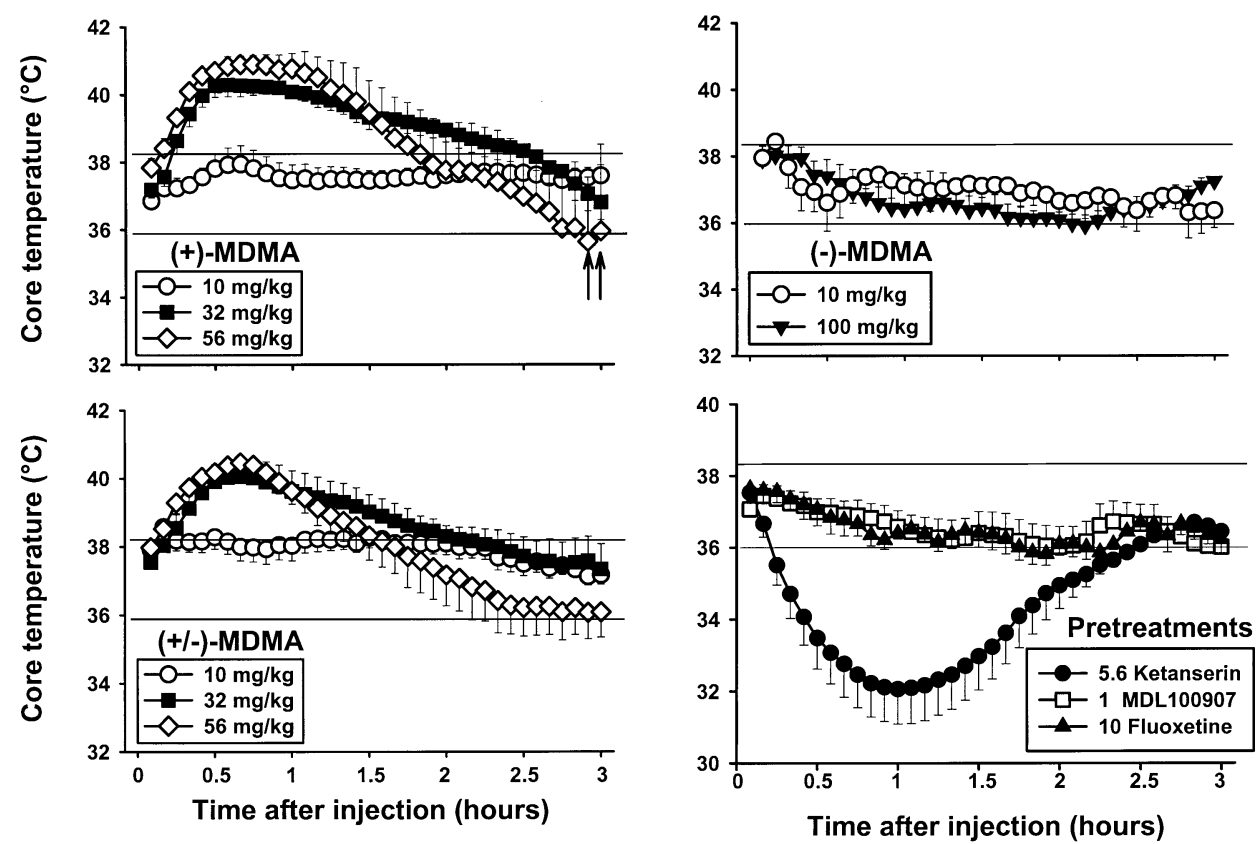
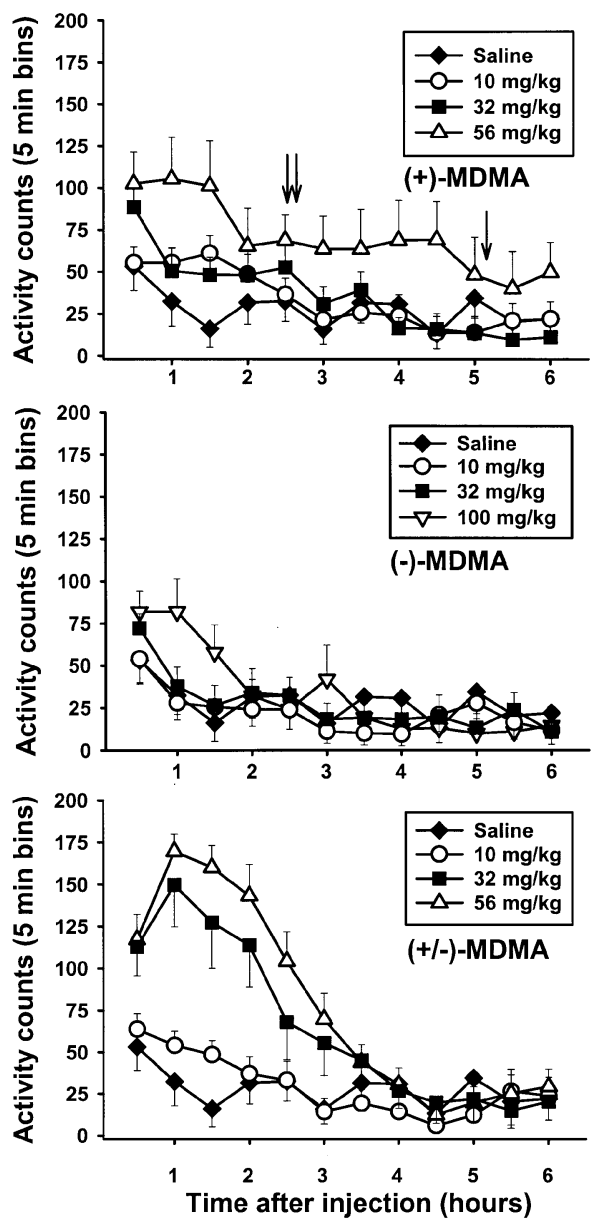

the remainder of the 24-h sampling period (data not shown).

Racemic MDMA and its stereoisomers each produced a long-lasting and dose-dependent stimulation of locomotor activity (Fig. 4). Racemic MDMA was most potent in inducing hyperactivity, producing a significant stimulation of locomotor activity during the first $15 \mathrm{~min}$ following injection of $10 \mathrm{mg} / \mathrm{kg}$. Racemic MDMA was also most effective within this behavioral measure, producing a peak of 175 activity counts at a dose of $56 \mathrm{mg} / \mathrm{kg}$; this stimulation of locomotor activity lasted approximately $3.5 \mathrm{~h}$. As with the core temperature experiments, $S(+)$-MDMA was approximately equipotent with racemic MDMA, producing a significant stimulation of locomotor activity at 10,15 , and $20 \mathrm{~min}$ following injection of $10 \mathrm{mg} / \mathrm{kg}$. The maximal number of activity counts collected following $S(+)$-MDMA administration was approximately 100 at a dose of $56 \mathrm{mg} / \mathrm{kg}$. Although of lesser magnitude than the stimulation produced by racemic MDMA, the hyperactivity induced by $S(+)-$ MDMA was longer lasting, returning to basal levels approximately $5 \mathrm{~h}$ post-injection. This dose produced several deaths (indicated by the arrows on Fig. 4, top panel), which precluded the testing of higher doses. $R(-)-$ MDMA was tenfold less potent than racemic or $S(+)$ MDMA, producing a significant stimulation of locomotor

Fig. 4 Effects of racemic methylenedioxymethamphetamine (MDMA) and its enantiomers on locomotor activity in singly housed mice. Each point represents the mean \pm SEM ( $n=6$ mice per dose). Abscissae Time after injection (h). Ordinates Activity counts (measured via implanted radiotelemetry probe). Arrows in top panel indicate time of death of one mouse per arrow following injection of $56 \mathrm{mg} / \mathrm{kg} S(+)$-MDMA 
Fig. 5 Effects of ketanserin pretreatments on $S(+)$ methylenedioxymethamphetamine (MDMA)- (left) and racemic MDMA- (right) induced hyperthermia (top) and locomotor stimulation (bottom). Each point represents the mean \pm SEM ( $n=6$ mice per dose). Abscissae and ordinates are as previously described in Fig. 3 (temperature) and Fig. 4 (activity)

Fig. 6 Effects of MDL100907 pretreatments on $S(+)-$ methylenedioxymethamphetamine (MDMA)- (left) and racemic MDMA- (right) induced hyperthermia (top) and locomotor stimulation (bottom). Each point represents the mean \pm SEM $(n=6$ mice per dose). Abscissae and ordinates are as previously described in Fig. 3 (temperature) and Fig. 4 (activity)
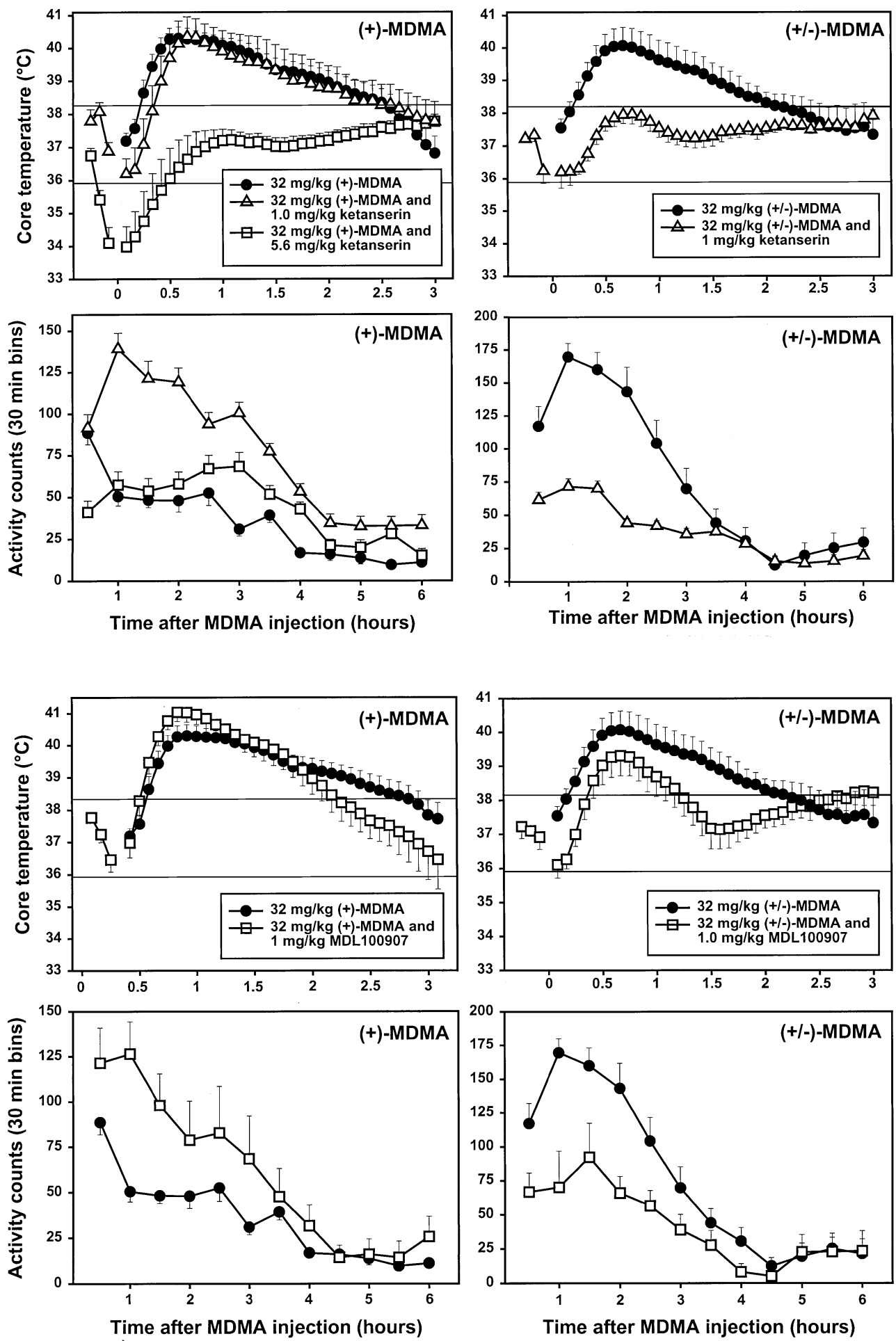

activity only during the first 15 min following administration of $100 \mathrm{mg} / \mathrm{kg}$, the highest dose tested. $R(-)$ MDMA was also the least effective within this behavioral measure, producing a maximum of approximately 75 activity counts at the highest dose administered. The locomotor stimulation produced by $R(-)$-MDMA was also of the shortest duration, returning to basal levels approximately $2 \mathrm{~h}$ post-injection. Neither ketanserin,
MDL100907, nor fluoxetine had any significant effects on locomotor activity relative to equivolume saline controls at the doses tested (data not shown).

When administered as a pretreatment for $S(+)$-MDMA, $1 \mathrm{mg} / \mathrm{kg}$ ketanserin was ineffective in blocking the induction of hyperthermia. S(+)-MDMA-induced hyperthermia was, however, blocked by $5.6 \mathrm{mg} / \mathrm{kg}$ ketanserin, although the direct effects of this dose of ketanserin on 
Fig. 7 Effects of fluoxetine pretreatments on $S(+)$ methylenedioxymethamphetamine (MDMA)- (left) and racemic MDMA- (right) induced hyperthermia (top) and locomotor stimulation (bottom). Each point represents the mean \pm SEM $(n=6$ mice per dose). Abscissae and ordinates are as previously described in Fig. 3 (temperature) and Fig. 4 (activity)
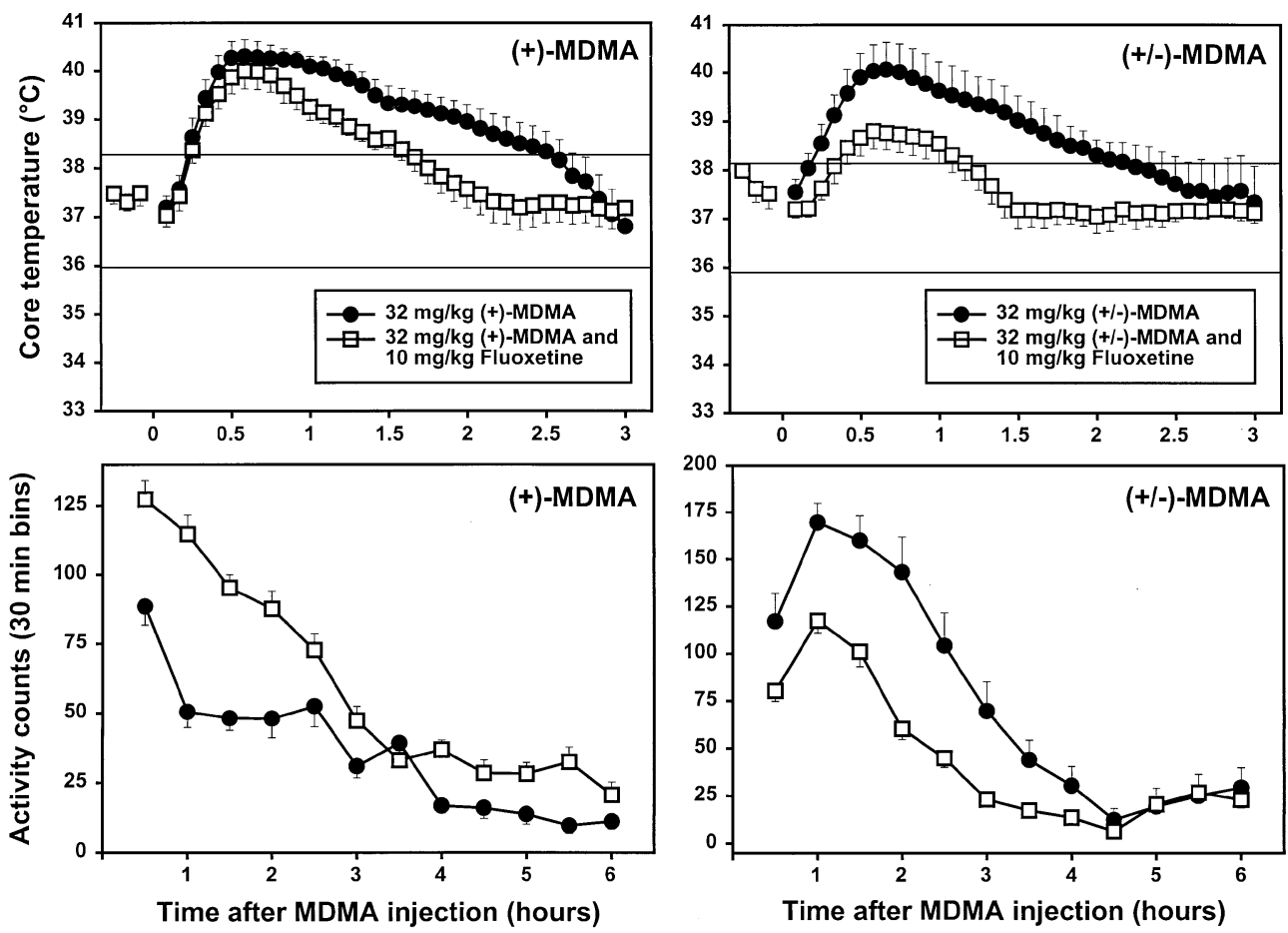

core temperature may be involved in this action. At a dose of $5.6 \mathrm{mg} / \mathrm{kg}$, ketanserin significantly decreased core temperature during the pretreatment time but did not prevent the subsequent $S(+)$-MDMA injection from rapidly increasing core temperature (Fig. 5, top left panel).

In contrast, $1 \mathrm{mg} / \mathrm{kg}$ ketanserin completely blocked the hyperthermic (Fig. 5, top right panel) and locomotor stimulant effects (Fig. 5, bottom right panel) of racemic MDMA without altering either core temperature or locomotor activity on its own. Interestingly, ketanserin pretreatments increased the maximal locomotor counts observed following $S(+)$-MDMA injections, as well as the duration of the locomotor stimulant effects. This effect was particularly pronounced at $1 \mathrm{mg} / \mathrm{kg}$ (Fig. 5 , bottom left panel).

Pretreatment with $1 \mathrm{mg} / \mathrm{kg}$ MDL100907 did not block the induction of $S(+)$-MDMA-induced hyperthermia (Fig. 6, top left panel). However, this dose of MDL100907 significantly attenuated both the hyperthermic (Fig. 6, top right panel) and locomotor stimulant (Fig. 6, bottom right panel) effects of racemic MDMA. As previously observed with the ketanserin pretreatments, MDL10097 also potentiated the locomotor stimulant effects of $S(+)$-MDMA, both in terms of magnitude and duration (Fig. 6, bottom left panel).

When administered as a pretreatment for $S(+)$-MDMA, $10 \mathrm{mg} / \mathrm{kg}$ fluoxetine was ineffective in blocking the induction of hyperthermia, although the duration of this effect was reduced (Fig. 7, top left panel). In contrast, fluoxetine significantly attenuated the hyperthermic (Fig. 7, top right panel) and locomotor stimulant (Fig. 7, bottom right panel) effects of racemic MDMA. As was the case for the other pretreatments, fluoxetine also potentiated $S(+)$-MDMA-induced locomotor stimulation (Fig. 7, bottom left panel) both in terms of magnitude and duration of effect.

\section{Discussion}

The neuropharmacology of MDMA is quite complex, involving interactions with pre- and postsynaptic receptors across several neurotransmitter systems. In rodents, MDMA primarily causes a presynaptic release of 5-HT (Rudnick and Wall 1992), although dopamine and norepinephrine are also released in lesser amounts, possibly via a similar transporter-mediated effect (Schmidt et al. 1987; Rothman et al. 2001). Additionally, MDMA has affinity for $5-\mathrm{HT}_{2}$ receptors, as well as histamine $\mathrm{H}_{1}$ receptors and muscarinic $\mathrm{M}_{1}$ receptors (Battaglia et al. 1988), and has recently been shown to act as an agonist at the rat trace amine receptor TAR1 (Bunzow et al. 2001). Finally, recent evidence has shown that MDMA induces acetylcholine release, both from rat striatal slices in vitro (Fischer et al. 2001) and as measured in vivo in rat prefrontal cortex and striatum by microdialysis (Acquas et al. 2001). This convoluted cluster of effects is further obfuscated by the fact that many of these neurochemical effects are linked, leading Parrot to describe this body of neuropharmacological research as "a minefield of potential drug interactions" (Parrot 2001).

This complicated neuropharmacology is due in part to the fact that racemic MDMA is made up of two active isomers, each with its own distinct bundle of effects that 
are distinguished not only in terms of quantitative differences, but also in terms of qualitative differences. Relatively few studies have directly compared the MDMA enantiomers across measures, which makes characterizing the isomers difficult. However, it is becoming readily apparent that the isomers have heterogeneous effects that manifest themselves across a wide range of endpoints, perhaps indicating important differences in their underlying pharmacology.

In this regard, the dissociation of the lethal effects of racemic MDMA and its isomers across housing conditions is especially intriguing. Ex vivo radioligand binding experiments have shown that $R(-)$-MDMA has greater affinity for 5- $\mathrm{HT}_{2}$ receptors than does $S(+)$-MDMA (Battaglia and De Souza 1989). Additionally, we have recently shown that ketanserin and MDL100907 both selectively attenuate the reinforcing effects of MDMA and its isomers in rhesus monkeys, and that $R(-)$-MDMAmaintained behavior is more susceptible to this antagonism than is behavior maintained by racemic or $S(+)$ MDMA (Fantegrossi et al. 2002). However, in the present experiments, MDL100907 was most protective against $R(-)$-MDMA-induced lethality in singly housed mice, while ketanserin pretreatments blocked $S(+)$-MDMAinduced lethality much more effectively than $R(-)$ MDMA-induced lethality. These findings clearly complicate a $5-\mathrm{HT}_{2}$ based interpretation of the present lethality data. Similarly, although $S(+)$-MDMA has previously been shown to possess higher affinity for 5-HT transporters than $R(-)$-MDMA (Battaglia and De Souza 1989), neither enantiomer was sensitive to fluoxetine pretreatment in any housing condition. Thus, these data, and the results of the cold-room protection experiments, may be better understood in the context of core temperature.

The present experiments using radiotelemetry probes demonstrate that racemic and $S(+)$-MDMA produce hyperthermia in singly housed mice at sublethal doses. In this regard, the complete protection afforded against the lethal effects of racemic and $S(+)$-MDMA in singly housed and crowded mice in the ketanserin antagonism experiments may be due to ketanserin-induced hypothermia. Similarly, induction of a hypothermic state via reduction of the ambient temperature to $4^{\circ} \mathrm{C}$ may be responsible for the complete abolition of the lethal effects of racemic and $S(+)$-MDMA in crowded mice and attenuation of the lethal effects of racemic MDMA in singly housed animals. This would imply that the lethal effects of racemic and $S(+)$-MDMA are closely related to their hyperthermic effects, especially in crowded settings. However, the protection afforded by ketanserin was much greater than that afforded by the $4^{\circ} \mathrm{C}$ ambient temperature for $S(+)$-MDMA-induced lethality, perhaps due to a lesser degree of hypothermia induced by the ambient temperature manipulation than by ketanserin.

In contrast, $R(-)$-MDMA does not increase core temperature even at doses with measurable lethal effects. Since $R(-)$-MDMA does not produce hyperthermia, ambient temperature manipulation affords no protection against the lethal effects of this compound in any housing condition. Similarly, induction of a hypothermic state via ketanserin pretreatment is not effective in protecting against the lethal effects of $R(-)$-MDMA in singly housed animals, although some protection is afforded to crowded mice. This may imply a temperature-dependent component to $R(-)$-MDMA-induced lethality in aggregate conditions.

Since neither MDL100907 nor fluoxetine induced hypothermia at the doses tested, their observed protection against the lethal effects of racemic MDMA may be related to their ability to block the induction of hyperthermia following injection of this compound. Both MDL100907 and fluoxetine significantly attenuated the hyperthermic effects of racemic MDMA in singly housed animals, and these pretreatments also proved effective in reducing lethality under this caging condition. Consistent with this explanation is the observation that these pretreatments were not effective in blocking the hyperthermic effects of $S(+)$-MDMA. The failure of MDL100907 and fluoxetine to protect against the lethal effects of $S(+)$-MDMA in singly housed mice is thus understandable in this context.

However, the protection afforded against $R(-)$ MDMA-induced lethality by MDL100907 in the singly housed condition cannot be explained by invoking arguments based on core temperature, for, if this were the case, one would expect to also attenuate the lethal effects of $R(-)$-MDMA with the hypothermic dose of ketanserin or by lowering the ambient temperature to $4^{\circ} \mathrm{C}$. The selective effects of MDL100907 against $R(-)$ MDMA-induced lethality are thus unique in that this is the only instance in which a pretreatment was effective in the singly housed condition but not in the aggregate condition. For $S(+)$-MDMA and the racemate, all manipulations were more effective at blocking lethality in crowded conditions, and this was also the case with $R(-)$ MDMA when ketanserin was administered as a pretreatment. Thus, this effect is likely a property of MDL100907 not $R(-)$-MDMA. The antagonist effects of MDL100907 against $R(-)$-MDMA-induced lethality in singly housed animals would seem to warrant further study.

The present results also indicate that the effects of racemic MDMA are quite different than one would expect based on the additive effects of the enantiomers. For example, the locomotor stimulation produced by racemic MDMA in the present study is of a larger magnitude than would be predicted by simply adding the effects of the individual isomers. Likewise, since $R(-)$-MDMA does not increase core temperature, racemic MDMA should be only half as potent as $S(+)$-MDMA in terms of hyperthermic effects. Such was not the case in the present studies where $S(+)$-MDMA and racemic MDMA were virtually equipotent, echoing the similar "disconcerting absence of additivity between the component isomers in comparison with the activity of the racemate", noted previously in studies of MDMA and its enantiomers in rabbit thermoregulation and human intoxication (Anderson et al. 1978). Finally, since the lethal effects of $R(-)$ MDMA were not altered by ketanserin pretreatment or by 
lowering the ambient temperature, the demonstrated protective effects of these manipulations should be approximately half as pronounced for racemic MDMA as for $S(+)$-MDMA. Paradoxically, in singly housed animals, ketanserin pretreatment proved equally effective against the lethal effects of $S(+)$-MDMA and the racemate, while the $4^{\circ} \mathrm{C}$ cold room proved protective only against the lethal effects of racemic MDMA. These findings seem to imply that the MDMA enantiomers somehow synergize in vivo to mediate the effects of racemic MDMA. This possibility deserves further study in order to elucidate the potential mechanisms underlying these complex findings.

Although quite similar in terms of their actions on core temperature, the hyperthermic effects of racemic MDMA and its $S(+)$ enantiomer can be easily distinguished when challenged with the various pretreatments employed in the present studies. A generalization of the results from all three pretreatment drugs suggests that the hyperthermic effects of racemic MDMA are sensitive to serotonergic manipulation, while $S(+)$-MDMA-induced hyperthermia is relatively resistant to these various pretreatment effects. The hyperthermic effects of racemic MDMA were completely blocked by a dose of ketanserin that did not induce hypothermia on its own, yet this ketanserin dose had no effect on $S(+)$-MDMA-induced hyperthermia. Similarly, doses of MDL100907 and fluoxetine that attenuated the hyperthermic effects of racemic MDMA did not alter the hyperthermic effects of $S(+)$-MDMA.

This pattern of interaction is in sharp contrast to that observed with the locomotor activity data. As with the effects on core temperature, all pretreatments significantly attenuated the locomotor stimulant effects of racemic MDMA. However, these same pretreatments all produced a significant potentiation of the locomotor stimulant effects of $S(+)$-MDMA, suggesting that the locomotor stimulant effects of racemic and $S(+)$-MDMA are mediated via distinct pharmacological mechanisms. Potentiated locomotor stimulant effects of MDMA have previously been observed following pretreatment with the nonselective $5-\mathrm{HT}_{1 / 2}$ antagonist methysergide (Gold and Koob 1998) or the 5- $\mathrm{HT}_{2 \mathrm{~B} / 2 \mathrm{C}}$ antagonist SB 206553 (Bankson and Cunningham 2001). However, since the effects of methysergide were assessed only in combination with racemic MDMA, and since the effects of SB 206553 were studied only in combination with $S(+)$ MDMA, it is difficult to interpret these data in terms of an overarching theory on the potential pharmacological mechanisms of MDMA-induced hyperactivity. A direct comparison of the isomers, ideally across a wide range of doses, seems critical in elucidating the unique pharmacology of the locomotor stimulant effects of the MDMA compounds.

Acute MDMA exposure has been linked to toxicity and lethality in humans, and these effects seem to occur most frequently following MDMA administration in "rave" settings (Henry et al. 1992; Randall 1992). This apparent potentiation of the lethal effects of MDMA in humans following ingestion of the drug in crowded settings may be analogous to the aggregate toxicity of MDMA and its enantiomers quantified by the present experiments. The influence of crowding may extend to MDMA effects other than lethality. Aggregate toxicity methodologies might therefore be applied to studies on the neurotoxicity produced by MDMA following acute and chronic dosing regimens to better model the environment in which the drug is often administered by humans.

The preponderance of evidence linking the hyperthermic effects of $S(+)$-MDMA to neurodegeneration in mice has been reviewed previously (Miller and O'Callaghan 1995); however, to our knowledge, similarly extensive studies have not been conducted with $R(-)$-MDMA. Given the presently demonstrated inability of $R(-)$ MDMA to induce hyperthermia in mice, one may predict that this enantiomer would lack neurotoxic effects, and there is some evidence that this may be the case in rats. Schmidt (1987) found that $10 \mathrm{mg} / \mathrm{kg}$ and $20 \mathrm{mg} / \mathrm{kg} S(+)-$ MDMA dose dependently reduced cortical 5-HT concentrations in rats 1 week after drug administration, and that the $20-\mathrm{mg} / \mathrm{kg}$ dose also produced an approximate $50 \%$ reduction in $\left[{ }^{3} \mathrm{H}\right] 5-\mathrm{HT}$ uptake. In contrast, neither $10 \mathrm{mg} /$ $\mathrm{kg}$ nor $20 \mathrm{mg} / \mathrm{kg} R(-)$-MDMA had any effect on these measures 1 week after administration (Schmidt 1987). However, given the potency differences here described for the lethal, core temperature, and locomotor stimulant effects of the MDMA enantiomers, it does seem possible that the $R(-)$-MDMA doses used in the above study were simply too low to produce similar neurochemical effects to the $S(+)$ enantiomer. The widely accepted relationship between MDMA-induced neurodegeneration and hyperthermia could therefore be strengthened or challenged by more fully assessing the neurotoxic potential of $R(-)$ MDMA across a wider dose range, and across caging conditions.

The present report provides evidence that the environment in which MDMA is administered has large interactive effects with MDMA-induced lethality, clearly highlighting the increased risk of ingesting MDMA in club and rave settings. The findings that exposure to a cold ambient environment and various serotonergic manipulations are capable of attenuating the lethal effects of racemic and $S(+)$-MDMA when administered in crowded conditions provide support for current treatments for acute MDMA-induced hyperthermia; however, the relative inability of these manipulations to protect against the lethal effects of $R(-)$-MDMA may pose a challenge to these and future potential therapeutics. Additionally, the dissociation of the lethal, hyperthermic, and locomotor stimulant effects of racemic and $S(+)$-MDMA argue that these compounds may have distinct pharmacological effects, and caution against using these drugs interchangeably in experiments investigating neurotoxicity and temperature. The challenge of unraveling the unique effects of MDMA on physiology and behavior is not likely to be overcome without further research into the distinct pharmacology of the MDMA enantiomers. 
Acknowledgements These studies were supported by USPHS grants DA09161 and DA05923. The authors express their gratitude for the expert technical assistance provided by Jon Dunker, Hosani McPherson, and the University of Michigan Unit for Laboratory Animal Medicine staff.

\section{References}

Acquas E, Marrocu P, Pisanu A, Cadoni C, Zernig G, Saria A, DiChiara G (2001) Intravenous administration of ecstasy (3,4methylenedioxymethamphetamine) enhances cortical and striatal acetylcholine release in vivo. Eur J Pharmacol 418:207211

Anderson GM III, Braun G, Braun U, Nichols DE, Shulgin AT (1978) Absolute configuration and psychotomimetic activity. NIDA Res Monogr 22:8-15

Bankson MG, Cunningham KA (2001) Pharmacological studies of the acute effects of (+)-3,4-methylenedioxymethamphetamine on locomotor activity: role of 5- $\mathrm{HT}_{2 \mathrm{~B} / 1 \mathrm{D}}$ and $5-\mathrm{HT}_{2}$ receptors. Neuropsychopharmacology 26:40-52

Battaglia G, Brooks B, Kulsakdinum C, DeSouza E (1988) Pharmacologic profile of MDMA (3,4-methylenedioxymethamphetamine) at various brain recognition sites. Eur J Pharmacol 149:159-163

Battaglia G, DeSouza E (1989) Pharmacologic profile of amphetamine derivatives at various brain recognition sites: selective effects on serotonin systems. NIDA Res Monogr 94:240-258

Broening H, Bowyer J, Slikker W (1995) Age dependent sensitivity of rats to the long term effects of the serotonergic neurotoxicant (+/-)-3,4-methylenedioxymethamphetamine (MDMA) correlates with the magnitude of the MDMA-induced thermal response. J Pharmacol Exp Ther 275:325-333

Bunzow JR, Sonders MS, Arttamangkul S, Harrison LM, Zhang G, Quigley DI, Darland T, Suchland KL, Pasumamula S, Kennedy JL, Olson SB, Magenis RE, Amara SG, Grandy DK (2001) Amphetamine, 3,4-methylenedioxymethamphetamine, lysergic acid diethylamide, and metabolites of the catecholamine neurotransmitters are agonists of a rat trace amine receptor. Mol Pharmacol 60:1181-1188

Chance M (1947) Aggregation as a factor influencing the toxicity of sympathomimetic amines in mice. J Pharmacol Exp Ther 87:214-219

Colado M, Granados R, O'Shea E, Esteban B, Green A (1998) Role of hyperthermia in the protective action of clomethiazole against MDMA ('ecstasy')-induced neurodegeneration, comparison with the novel NMDA channel blocker AR-R15896AR. Br J Pharmacol 124:479-484

Davis W, Borne R (1984) Pharmacologic investigation of compounds related to 3,4-methylenedioxyamphetamine (MDA). Subst Alcohol Actions/Misuse 5:105-110

Doggett N, Reno H, Spencer P (1977) A comparison of the acute toxicity of some centrally acting drugs measured under crowded and uncrowded conditions. Toxicol Appl Pharmacol 39:141-148

Fantegrossi W, Ullrich T, Rice K, Woods J, Winger G (2002) 3,4methylenedioxymethamphetamine (MDMA, 'Ecstasy') and its stereoisomers as reinforcers in rhesus monkeys: serotonergic involvement. Psychopharmacology 161:356-364

Farfel G, Seiden L (1995) Role of hypothermia in the mechanism of protection against serotonergic toxicity. I. Experiments with 3,4-methylenedioxymethamphetamine, dizocilpine, CGS 19755 and NBQX. J Pharmacol Exp Ther 272:868-975

Fischer H, Zernig G, Schatz D, Humpel C, Saria A (2001) MDMA (ecstasy) enhances basal acetylcholine release in brain slices of the rat striatum. Eur J Neurosci 12:1385-1390

Gold LH, Koob GF (1988) Methysergide potentiates the hyperactivity produced by MDMA in rats. Pharmacol Biochem Behav 29:645-648
Green A, Cross A, Goodwin G (1995) Review of the pharmacology and clinical pharmacology of 3,4-methylenedioxymethamphetamine (MDMA or "Ecstasy"). Psychopharmacology 119:247-260

Greenblatt E, Osterberg A (1961) Correlations of activating and lethal effects of excitatory drugs in grouped and isolated mice. J Pharmacol Exp Ther 131:115-119

Gunn J, Gurd M (1940) The action of some amines related to adrenaline. Cyclohexylalkylamines. J Physiol 97:453-470

Henry J, Jeffreys K, Dawling S (1992) Toxicity and deaths from 3,4-methylenedioxymethamphetamine ("ecstasy"). Lancet 340:384-387

Malberg J, Seiden L (1998) Small changes in ambient temperature cause large changes in 3,4-methylenedioxymethamphetamine (MDMA)-induced serotonin neurotoxicity and core body temperature in the rat. J Neurosci 18:5086-5094

Malberg J, Sabol K, Seiden L (1996) Co-administration of MDMA with drugs that protect against MDMA neurotoxicity produces different effects on body temperature in the rat. J Pharmacol Exp Ther 278:258-267

Mazzola-Pomieto P, Aulakh C, Tolliver T, Murphy D (1997) Functional subsensitivity to $5 \mathrm{HT}_{2} \mathrm{~A}$ and $5 \mathrm{HT}_{2 \mathrm{C}}$ receptors mediating hyperthermia following acute and chronic treatment with $5 \mathrm{HT}_{2 \mathrm{~A} / 2 \mathrm{C}}$ receptor agonists. Psychopharmacology 130:144-151

Miller D, O'Callaghan J (1994) Environment-, drug- and stressinduced alterations in body temperature affect the neurotoxicity of substituted amphetamines in the C57BL/6 J mouse. J Pharmacol Exp Ther 270:752-760

Miller D, O'Callaghan J (1995) The role of temperature, stress, and other factors in the neurotoxicity of the substituted amphetamines 3,4-methylenedioxymethamphetamine and fenfluramine. Mol Neurobiol 11:177-192

Mohrland J, Craigmill A (1980) Possible mechanism for the enhanced lethality of morphine in aggregated mice. Pharmacol Biochem Behav 13:475-477

Nash J, Meltzer H, Gudelsky G (1988) Elevation of serum prolactin and corticosterone concentrations in the rat after the administration of 3,4-methylenedioxymethamphetamine. J Pharmacol Exp Ther 245:873-879

Nash J, Roth B, Brodki J, Nichols D, Gudelsky G (1994) Effect of the $\mathrm{R}(-)$ and $\mathrm{S}(+)$ isomers of MDA and MDMA on phosphotidylinositol turnover in cultured cells expressing $5 \mathrm{HT}_{2 \mathrm{~A}}$ and $5 \mathrm{HT}_{2 \mathrm{C}}$ receptors. Neurosci Lett 177:111-115

Parrot A (2001) Human psychopharmacology of Ecstasy (MDMA): a review of 15 years of empirical research. Hum Psychopharmacol Clin Exp 16:557-577

Randall T (1992) Ecstasy-fueled 'rave' parties become dances of death for English youths. JAMA 268:1505-1506

Rothman R, Baumann M, Dersch C, Romero D, Rice K, Carroll F, Partilla J (2001) Amphetamine-type central nervous system stimulants release norepinephrine more potently than they release dopamine and serotonin. Synapse 39:32-41

Rudnick G, Wall S (1992) The molecular mechanism of $[3,4$ methylenedioxy-methamphetamine (MDMA)]: serotonin transporters are targets for MDMA-induced serotonin release. Proc Natl Acad Sci USA 89:1817-1821

Schmidt CJ (1987) Neurotoxicity of the psychedelic amphetamine methylenedioxymethamphetamine. J Pharmacol Exp Ther 240:1-7

Schmidt C, Levin J, Lovenberg W (1987) In vivo and in vitro neurochemical effects of methylenedioxymethamphetamine on striatal monoaminergic systems in the rat brain. Biochem Pharmacol 36:747-755

Ullrich T, Fantegrossi W, McMahon L, Cunningham K, Woods J, Rice K (2000) New chemical and biological studies on MDL100907, a highly selective serotonin 5- $\mathrm{HT}_{2 \mathrm{~A}}$ antagonist. College on Problems of Drug Dependence Abstracts, SixtySecond Annual Scientific Meeting, San Juan, Puerto Rico 